S ince early spring, the COVID-19 pandemic has catapulted the healthcare community from a largely comprehensible world of work to unfamiliar territory, where the ground is ever shifting. What does it take to serve the public in this environment? Constant recalibration, endless reserves of energy, quick wits and a wide-open mind willing to look for solutions in new places working with new people.

COVID-19 brought about swift change and challenges of a size and severity never known. The opening articles of this issue's Healthcare Quarterly suggest that the healthcare system can make change happen too, even if not overnight, but swifter still than healthcare's usual halting pace.

\section{Responding to the COVID-19 Pandemic}

Wojtak and colleagues (2020) tell a thorough, seamless story of the comprehensive, community-wide response that East Toronto Health Partners (ETHP) delivered to clearly identified populations from the pandemic's earliest days. They also provide an analytic account of the factors fuelling that response and reflect on how managing a complex response to COVID-19 has opened up possibilities for precipitating a larger system change.

As one of the first Ontario Health Teams, ETHP could draw on its recently honed skills and success in integrating a 50-member network of healthcare and social services organizations. ETHP became a cohesive entity through the hard work of integration - relationship and trust building, shared leadership across sectors and more. It was well positioned to provide a rapid and targeted response to a mix of neighbourhoods with varied needs. The "game changer" in responding to COVID-19 was the inclusion of organized primary care leadership at a local level.

Martin (2020) advises hospital boards to avoid a leadership vacuum regardless of the phase through which a pandemic is passing. While acknowledging the need for adjustments and flexibility, she underscores the importance of re-establishing the processes and tenets of good governance and reaffirming its principles without undue delays. Ensuring that board members abide by the rules and understand and fulfill their roles and responsibilities in meeting the needs of management, the hospital and the public is more critical during uncertain times.

The COVID-19 pandemic has generated many unknowns, but its disproportionate impact on at-risk populations is increasingly well documented. The article by Bhatti and colleagues (2020) is based on interviews with 70 organizations from the Alliance for Healthier Communities, whose members' COVID-19 responses reflect bedrock beliefs in principles such as equity and a commitment to protect at-risk populations. The authors provide a close-up of the care and support provided over the first three weeks of the pandemic, when services actually increased. During lockdown, all members replaced in-person services with virtual ones. When food banks closed, some provided grocery cards, delivered hot meals and tents and conducted wellness checks to identify those most at risk. Others reached out to help seniors and people experiencing homelessness, whether on or off their roster. The Alliance says that the strength of its early response shows the benefits of the salary-based funding model and systems-thinking approach embedded in community health centres.

\section{Innovation in Health Services}

O'Donnell et al. (2020) present "a pharmacist-driven medication reconciliation program during transitions to long-term care and retirement homes" as a cost-containment innovation to save system resources. The authors calculate that dedicating pharmacists to provide pretransition care services on a virtual basis will yield potential savings of $\$ 622.35$ per patient through averted hospitalizations and $\$ 1,414.52$ per patient per year from medication discontinuations. They also suggest that better patient outcomes will accrue from the program.

\section{Healthcare Governance}

Hooper (2020) notes that when performance management healthcare professionals embraced natural language processing tools as a method to quantify clinical quality, it was destined to reach hospital board tables. And that is a good thing, Hooper argues, because they can analyze board minutes to gain a better understanding of the board and a more precise measurement of its work. The tools can identify issues that have been overlooked or overemphasized so that boards can rebalance their time and attention to better align with priorities. Hooper sees great potential in the uptake of tools and techniques such as sentiment analysis and riverbed graphs, which he uses to analyze and present data from more than 700 meetings and 3,000 motions from Ontario hospital boards.

\section{Quality Improvement}

The most salient point in the article by El-Baba et al. (2020) is not the findings from the retrospective review examining wait times for patients visiting four Ontario community emergency departments (EDs) from 2016 to 2017 but rather what prompted the results. The study - the first to examine compliance with recommended Physician Initial Assessment times 
based on the Canadian Triage and Acuity Scale scores - reveals the EDs' low overall compliance with wait-time guidelines. The authors say better compliance is contingent on resolving systemic challenges and report that their analysis shows that the triage system does not influence the order of priority in which patients are seen and assessed. They recognize that ED wait times affect patient care but argue that poor scores reflect patient flow problems and inadequate downstream investments that cause overcrowding.

\section{Digital Health}

Given Ontario's persistent push toward integrated systems highly reliant on digital solutions and the ubiquity of stories about COVID-19 causing burnout among health providers, the article by Lo et al. (2020) is particularly on point. Drawing from a rapid literature review on the relationship between electronic health record (EHR) system usage and clinician burnout, the authors identify measures such as education and training, administrative support and the use of scribes as ways to reduce the stress and time-consuming tasks associated with EHR reliance.

\section{Persistent Healthcare Issues}

Positive changes aside, some ever-present healthcare issues are becoming more problematic. Institute for Clinical Evaluative Sciences data on Ontario's mental health and addictions system (2009 to 2017) show no real improvement in quality over time (Chiu et al. 2020). Youths aged 14-24 years experienced the highest rates of deliberate self-harm and the greatest rise over time in mental health and addictions-related outpatient and emergency department visits and hospitalizations. People with schizophrenia had some of the lowest access to care following discharge. The Canadian Institute for Health Information reports that the equivalent of 65 young people, aged 10 to 24, were hospitalized every day in Canada in 2017-2018 for harm caused by substance use. In $69 \%$ of these hospital stays, care for a mental health condition was included (Pichora et al. 2020).

\section{References}

Bhatti, S., E. Commisso and J. Rayner. 2020. A Rapid Primary Healthcare Response to COVID-19: An Equity-Based and SystemsThinking Approach to Care Ensuring that No One Is Left Behind. Healthcare Quarterly 23(3): 29-33. doi:10.12927/hcq.2020.26336..

Chiu, M., A. Guttmann and P. Kurdyak. 2020. Mental Health and Addictions System Performance in Ontario: An Updated Scorecard, 2009-2017. Healthcare Quarterly 23(3): 7-11. doi:10.12927/ hcq.2020.26340.

El-Baba, M., R. Ramos, L. Salehi, Q. Amin, S. Brown, S. Upadhye et al. 2020. Physician Initial Assessment Times Based on CTAS Scores: Are We Meeting the Recommendations? Healthcare Quarterly 23(3): 48-53. doi:10.12927/hcq.2020.26333.

Hooper, L. 2020. Measuring Boards Using Quantitative Tools from Natural Language Processing. Healthcare Quarterly 23(3): 41-7. doi:10.12927/hcq.2020.26334.

Lo, B., J. Kemp, C. Cullen, T. Tajirian, D. Jankowicz and G. Strudwick. 2020. Electronic Health Record-Related Burnout among Clinicians: Practical Recommendations for Canadian Healthcare Organizations. Healthcare Quarterly 23(3): 54-62. doi:10.12927/hcq.2020.26332.

Martin, N. 2020. Finding a New Normal: Good Governance Best Practices during COVID-19. Healthcare Quarterly 23(3): 24-8. doi:10.12927/hcq.2020.26337.

O’Donnell, D., C. Beaton, J. Liang, K. Basu, M. Hum, A. Propp, L. Yanni, Y. Chen and P. Ghafari. 2020. Cost Impact of a PharmacistDriven Medication Reconciliation Program during Transitions to Long-Term Care and Retirement Homes. 2020. Healthcare Quarterly 23(3): 34-40. doi:10.12927/hcq.2020.26335.

Pichora, E., A. Dudevich, G. Hynes and C. Catley. 2020. Hospital Stays for Harm Caused by Substance Use among Youth in Canada. Healthcare Quarterly 23(3): 12-14. doi:10.12927/hcq.2020.26339.

Wojtak, A., J. Altenberg, C. Annett, A. Babcock, K. Chung, S. Downey et al. 2020. Mobilizing a Whole Community: Policy and Strategy Implications of an Integrated Local System Response to a Global Health Crisis. Healthcare Quarterly 23(3): 15-23. doi:10.12927/ hcq. 2020.26338.

- The Editors 